\title{
Saudi License Plate Recognition
}

\author{
Saleh Basalamah
}

\begin{abstract}
Automatic license plate recognition systems (LPR) can help reduce the number of traffic violations and make our streets safer. In this project we developed an automatic system that locates Saudi license plates in a captured image regardless of the time of day or license plate scale. The proposed system can tolerate slight tilting of the license plate. The localization process is fairly complex due to the highly varying nature of the background. Good results were obtained using the localization stage. A second part of the system was developed to segment and recognize the characters in the located license plate.
\end{abstract}

Index Terms-Intelligent transportation systems, license plate recognition, saudi license plates.

\section{INTRODUCTION}

License plate recognition plays an important role in intelligent transportation systems. It serves applications in vehicle tracking, traffic violation, parking automation, etc...

The first to work on Saudi license plate recognition was Sarfraz [1]-[3] from KFUPM in 2003. These early works such as Sarfraz [1]-[3], Zidouri [4] and Khalil [5] were all on the previous Saudi license plates series.

Maglad [6], [7] proposed a system that uses radial basis function neural networks for the detection and recognition of plates. Khalid Almustafa [8]-[10] proposed an algorithm that uses pixel density to recognize characters in license plates. Shaaban [11] proposed a system where a neural network classifier is used to recognize the characters based on edge moment invariants and principal component analysis features of wavelet coefficient matrix.

Alginahi [12] proposed a system that recognized both old and new Saudi license plates. The license plates are detected by searching the vertical lines then the width to length ratio is used to locate the plate. Character segmentation is then performed before both a distance classifier and a Neural Network classifier are used to recognize characters.

Deriche [13] proposed a hybrid system of RFID and license plate recognition. The license plate detection is based on morphological filtering and the character recognition is based on neural networks. Mansour [14] proposed a system based on chain code and tested it on both Egyptian and Saudi license plates.

\section{SAudi License Plates}

The first Saudi license plates were introduced in the 1950s; a new plate series was introduced approximately every

Manuscript received October 18, 2012; revised November 27, 2012.

Saleh Basalamah is with the Computer Engineering Department, Umm Al-Qura University, Makkah, Saudi Arabia (e-mail: smbasalamah@uqu.edu.sa). decade. Figure (1) shows the five different Saudi plate series [15].

The current license plates have the following characteristics [16]:

- The plate size is $32 \mathrm{~cm} \times 16 \mathrm{~cm}$.

- The ratio of width to length is $2: 1$.

- The plate is divided into 5 sections; Arabic letters, Hindi numbers, Latin letters, Arabic numbers and vehicle type section which is color coded and includes the name and logo of Saudi Arabia.

- There are 4 color codes; white is for private cars, yellow is for transport cars, blue is for commercial cars and green is for diplomatic cars.

- Size of letters is $36 \times 21 \mathrm{~mm}$ and size of numbers is 55 $\times 26 \mathrm{~mm}$.

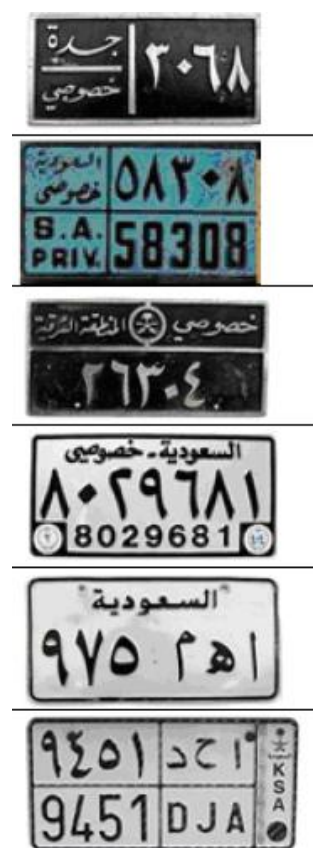

Fig. 1. History of Saudi license plates [15] introduced in (from top to bottom): 1950, 1962, 1972, 1978, 1996 and 2007.

\section{Proposed System}

\section{A. Localization}

Locating the license plate in an image is the first step in any license plate recognition system. Variation in license plate location in different cars, variation in location of cars in images and variation in lighting during day/night makes this step challenging.

We started looking into locating the plates by searching for black lines that borders the license plates thus we have to look for a horizontal line and a vertical line, however we 
found that in many cars that border is hidden under a frame that is commonly used to hold the plate.

We have noticed that particular to Saudi license plates, there is a black cross that centers all license plates, this cross is visible in all plates and is not hidden by frames since is it is in the center of the license plates.

We apply an edge detector to find the horizontal and vertical maps; we then use the average value of pixels in each map as a threshold to convert it into a binary image and use a median filter to remove noise and find the binary addition of the maps. To help remove incorrect candidates, we zero all ones that originate from a color region in the original image since the cross we are looking for is in a black and white plate.

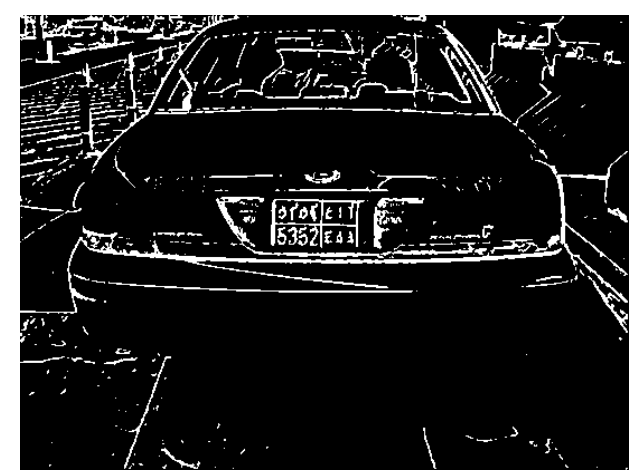

Fig. 2. Binary image after pre-processing to locate cross in the license plate.

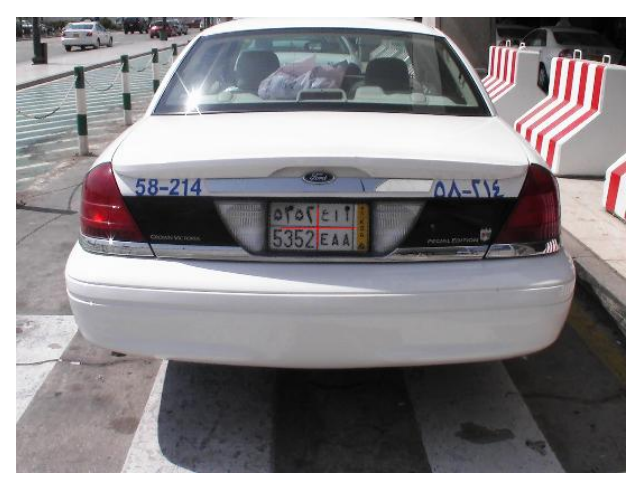

Fig. 3. Cross located in license plate.

This last binary image is finally searched for the cross and the center pixel is considered the center of the plate where the lowest difference is found.

The search for the cross could lead to false locations especially when there is slight rotation in the image. The search had to be modified to look for a black line within the neighborhood of 6 lines. This gives some tolerance to possible image rotation and overcomes the slight rotation problem. This also affects finding the exact license plate border and the image needs to be rotated slightly accordingly.

\section{B. Segmentation}

In order to perform the license plate recognition, our method segments the characters in the plates. The plates have to be aligned horizontally by finding the tilt angle that maximizes the sum of horizontal black lines into three areas, at the edges and at the middle. Figure (4) shows the horizontal line profile of a license plate with a tilt and shows that thresholded profile of an adjust tilt plate.
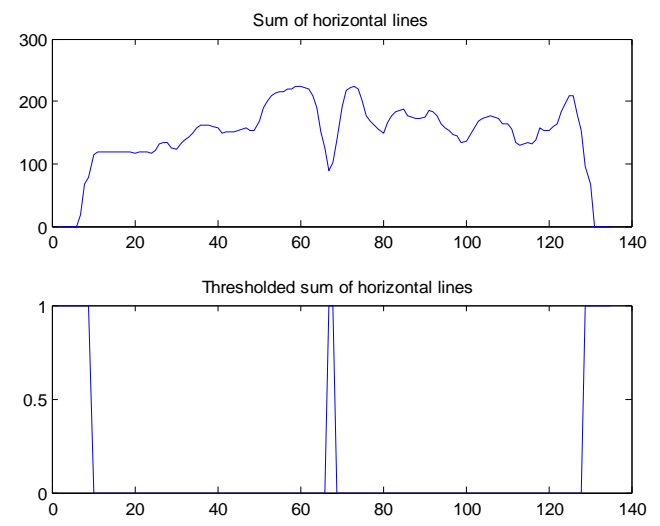

Fig. 4. Above is the profile of a tilted license plate and below is a thresholded profile of an adjusted license plate.

Once the license plate is horizontally aligned, several steps are then perfomed to segment the characters:

1) Cut the image horizontally in half.

2) Cut the lower image vertically in half.

3) Remove empty horizontal lines from both ends of lower left and lower right images.

4) Remove empty vertical lines from both ends of lower left and lower right images.

5) Segment numbers by moving left to right in the lower left image until finding empty vertical lines between numbers.

6) Segment letters by moving left to right in the lower right image until finding empty vertical lines between letters.

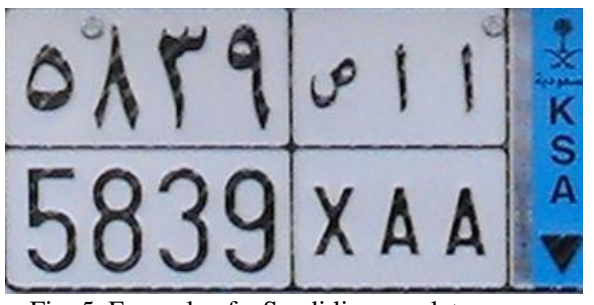

Fig. 5. Example of a Saudi license plate

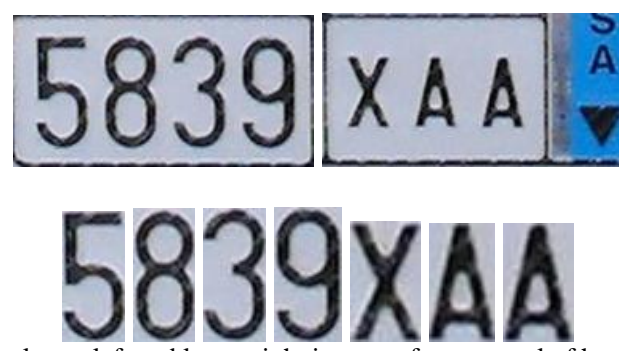

Fig. 6. The lower left and lower right images after removal of horizontal and vertical empty lines.

\section{Recognition}

After segmenting the numbers and letters in the license plate, these numbers and letters are recognized using a template matching technique and by comparing them to reference templates of all possible characters in Saudi license plates. The reference templates have been previously saved and organized. An image size of 50 x 50 pixels was also chosen for our reference templates. Since characters obtained from different license plate images have different sizes; all 
segmented characters have to be resized to $50 \times 50$ pixels. A simple difference comparison equation is used to compare the segmented characters to the reference templates.

$$
d=\sqrt{\left(x_{S e g}-x_{\operatorname{Re} f}\right)^{2}}
$$

Each segmented character is compared with all reference characters and it is recognized when it has the least difference value $d$.

\section{RESUlts}

The threshold value for creating the binary images was changed along with the median filter setting. This was done to find the settings that produce most correct plate centers in all the images. The following table shows a part of the full table that was produced to find the optimum values of the threshold multiplier and the median filter size.

\begin{tabular}{|c|c|c|c|c|c|c|c|c|c|c|c|c|c|c|c|c|c|c|}
\hline \multicolumn{19}{|c|}{ AND MEDIAN FILTER SETTINGS. } \\
\hline Multiplier & 1.25 & \multicolumn{5}{|c|}{1.5} & \multicolumn{5}{|c|}{1.75} & \multicolumn{5}{|c|}{2} & \multicolumn{2}{|c|}{2.25} \\
\hline Filter & 6 & 3 & 4 & 5 & 6 & 7 & 3 & 4 & 5 & 6 & 7 & 3 & 4 & 5 & 6 & 7 & 3 & 4 \\
\hline File Name & 32 & 11 & 12 & 13 & 14 & 15 & 16 & 17 & 18 & 19 & 20 & 21 & 22 & 23 & 24 & 25 & 26 & 27 \\
\hline PIC_0574 & 1 & 1 & 1 & 1 & 0 & 1 & 1 & 1 & 0 & 1 & 1 & 1 & 1 & 1 & 1 & 1 & 1 & 0 \\
\hline PIC_0575 & 1 & 1 & 1 & 1 & 1 & 1 & 1 & 1 & 1 & 1 & 1 & 1 & 1 & 1 & 1 & 1 & 1 & 1 \\
\hline PIC_0576 & 1 & 0 & 1 & 1 & 1 & 1 & 0 & 1 & 1 & 1 & 1 & 1 & 1 & 1 & 1 & 1 & 1 & 1 \\
\hline PIC_0577 & 0 & 0 & 0 & 0 & 0 & 0 & 0 & 0 & 0 & 0 & 0 & 0 & 0 & 0 & 0 & 0 & 0 & 0 \\
\hline PIC_0578 & 0 & 1 & 1 & 1 & 1 & 1 & 0 & 1 & 1 & 1 & 1 & 1 & 1 & 1 & 1 & 1 & 1 & 1 \\
\hline PIC_0579 & 1 & 0 & 0 & 0 & 0 & 0 & 0 & 0 & 0 & 0 & 0 & 0 & 0 & 0 & 0 & 0 & 0 & 0 \\
\hline PIC_0580 & 0 & 0 & 0 & 0 & 0 & 0 & 0 & 0 & 0 & 0 & 0 & 0 & 0 & 0 & 0 & 0 & 0 & 0 \\
\hline & & 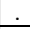 & . & . & . & . & . & . & . & . & . & . & . & . & . & . & . & . \\
\hline & . & . & . & . & . & . & . & . & . & . & & . & . & . & . & . & . & . \\
\hline & & & & & - & 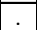 & 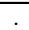 & 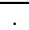 & 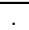 & 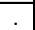 & & . & . & . & . & . & 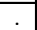 & . \\
\hline PIC_0655 & 1 & 1 & 1 & 1 & 1 & 1 & 1 & 1 & 1 & 1 & 1 & 1 & 1 & 1 & 1 & 1 & 1 & 1 \\
\hline PIC_0656 & 1 & 1 & 1 & 1 & 1 & 1 & 1 & 1 & 1 & 1 & 1 & 1 & 1 & 1 & 1 & 1 & 1 & 1 \\
\hline PIC_0657 & 1 & 0 & 0 & 0 & 0 & 0 & 0 & 0 & 0 & 0 & 1 & 0 & 0 & 0 & 0 & 0 & 0 & 0 \\
\hline PIC_0658 & 1 & 1 & 1 & 1 & 1 & 1 & 1 & 1 & 1 & 1 & 1 & 1 & 1 & 0 & 1 & 1 & 1 & 0 \\
\hline rcentage & $76.0 \%$ & 60 & 59 & 60 & 56 & 56 & 59 & 60 & 56 & 56 & 58 & 61 & 64 & 58 & 59 & 60 & 62 & 55 \\
\hline
\end{tabular}

The system was tested on 173 images and the localization method shows a success rate of $76 \%$ as can be seen in table (1). The segmentation method was successful on all license plates images that were correctly localized. The recognition method was correctly obtained in 140 images with a success rate of $81 \%$.

Results of the segmentation and recognition method are shown in figures (7-10)

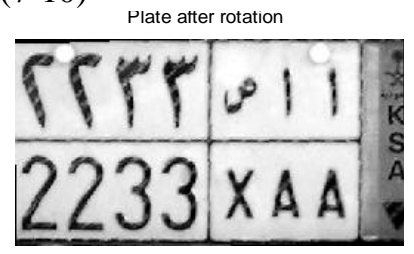

BW Plate after rotation

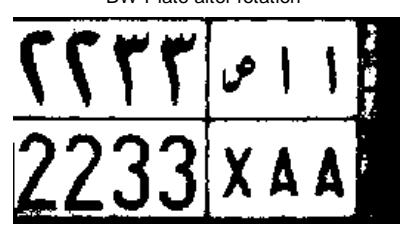

Fig. 7. Grayscale and binary images of license plate after tilt correction.

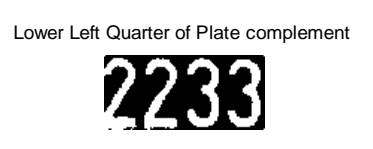

LLQ of Plate complement (empty lines removed)

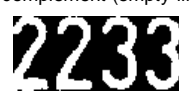

Lower Right Quarter of Plate complement

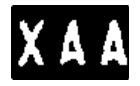

$\mathrm{LRQ}$ of Plate complement (empty lines removed)

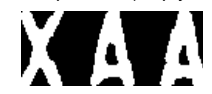

Fig. 8. Complemented lower left and lower right quarters after removal of empty horizontal lines.
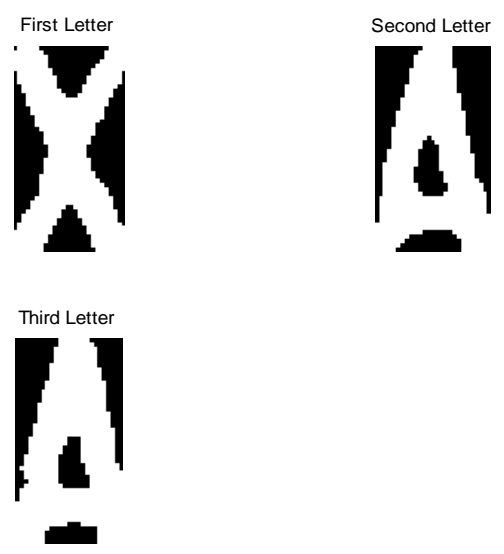

XAA

Fig. 9. The three letters segmented and recognized
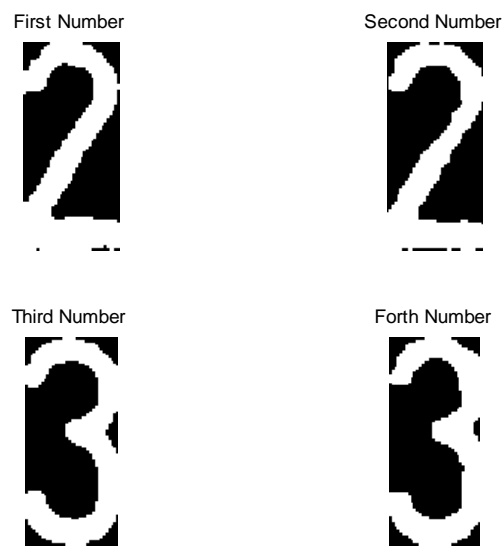

\section{3}

Fig. 10: The four numbers segmented and recognized

\section{CONCLUSION}

The proposed system locates and recognizes license plates under complex scenarios like variation of illumination, angle, and scale. Good results have been obtained. Further work can be added by using a more robust comparison method in the recognition part of the system to increase the accuracy of the recognition. The accuracy of the system is also expected to increase by mapping the recognition of the Arabic characters and the Latin characters together. 


\section{ACKNOWLEDGMENT}

The author would like to thank Raed Marghalani and Emad Gari for their great help in this work.

\section{REFERENCES}

[1] M. Sarfraz, M. J. Ahmed, and S. A. Ghazi, "Saudi Arabian license plate recognition system," in Proc. of International Conference on Geometric Modeling and Graphics, pp. 36-41, 2003.

[2] M. J. Ahmed, M. Sarfraz, A. Zidouri, and W. G. Al-Khatib, "License plate recognition system," in Proc. of 10th IEEE International Conference on Electronics, Circuits and Systems, pp. 898-901, 2003.

[3] M. Sarfraz and M. Jameel, "License plate recognition system: Saudi Arabian case," in Computer-Aided Intelligent Recognition Techniques and Applications, M. Sarfraz, Ed. Chichester, UK: John Wiley \& Sons, ch. 2, 2005.

[4] A. Zidouri and M. Deriche, "Recognition of Arabic license plates using NN," in First Workshops on Image Processing Theory, Tools and Applications, pp. 1-4, 2008.

[5] M. I. Khalil, "Car plate recognition using the template matching method," International Journal of Computer Theory and Engineering, vol. 2, no. 5, pp. 683-687, Oct. 2010.

[6] K. Maglad, D. Mohamad, and N. A. Abulgasem, "Saudian car license plate number detection and recognition using morphological operation and RBF neural network," Australian Journal of Basic and Applied Sciences, vol. 5, no. 12, pp. 1780-1786, Dec. 2011.

[7] K. W. Maglad, "A vehicle license plate detection and recognition system," in Journal of Computer Science, vol.8, no. 3, pp. 310-315, Jan. 2012.

[8] K. M. Almustafa, R. N. Zantout, and H. R. Obeid, "Pixel density: Recognizing characters in Saudi license plates," in Proc. 10th International Conference on Intelligent Systems Design and Applications, pp. 308-313, 2010.

[9] K. M. Almustafa, R. N. Zantout, and H. R. Obeid, "Peak position recognizing characters in Saudi license plates," in Proc. IEEE GCC Conference and Exhibition, Dubai, pp. 186-189, 2011.
[10] K. M. Almustafa, R. N. Zantout, H. R. Obeid, and F. Sibai, "Recognizing characters in Saudi license plates using character boundaries," in Proc. International Conference on Innovations in Information Technology, Abu Dhabi, pp. 415-420, 2011.

[11] Z. Shaaban, "An intelligent license plate recognition system," International Journal of Computer Science and Network Security, vol. 11, no. 7, Jul. 2011.

[12] Y. M. Alginahi, "Automatic Arabic License Plate Recognition," International Journal of Computer and Electrical Engineering, vol. 3, no. 3, pp. 454-460, pp. 55-61, Jun. 2011.

[13] M. Deriche and M. Mohandes, "A hybrid RFID-LPR system for vehicle access control during pilgrimage season in Saudi Arabia," in Proc. 9th International Multi-Conference on Systems, Signals and Devices, Chemnitz, pp. 1-6, 2012.

[14] R. F. Mansour, "A robust method for Arabic car plates recognition and matching using chain code," American Journal of Computational and Applied Mathematics, vol. 2, no. 3, pp. 105-111, 2012.

[15] A. M. El-Sherbeeny, H. M. Al Sohaibani, A. S. Al Sohaibani, and A. A. Al Sohaibani, "Evaluating the design of the current Saudi Arabian license plate," in Proc. of 41st International Conf. on Computers and Industrial Engineering, Los Angeles, pp. 223-234, 2011.

[16] A. S. Al Sohaibani, H. M. Al Sohaibani, and A. A. Al Sohaibani, "Redesign of Saudi License Plate," B. S. capstone project, Dept. Industrial Engineering, King Saud University, Riyadh, Saudi Arabia, 2011.

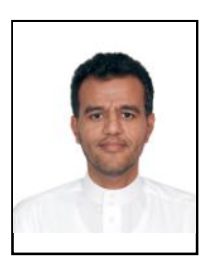

Saleh Basalamah is an assistant professor at Umm Al-Qura University. His research interests include computer vision, multimedia and information security. Saleh has a $\mathrm{PhD}$ in bioengineering from Imperial College London. He's a member of IEEE and ACM. Contact him at smbasalamah@uqu.edu.sa. 